\title{
Absence of the strahl during times of slow wind
}

\author{
Chris Gurgiolo $^{1}$ and Melvyn L. Goldstein ${ }^{2}$ \\ ${ }^{1}$ Bitterroot Basic Research, Hamilton, MT, USA \\ ${ }^{2}$ Heliospheric Physics Laboratory, Code 672, NASA Goddard Space Flight Center, Greenbelt, MD, USA
}

Correspondence to: Chris Gurgiolo (chris@gurgiolo.com)

Received: 17 June 2016 - Revised: 28 November 2016 - Accepted: 12 December 2016 - Published: 5 January 2017

\begin{abstract}
It is not uncommon during periods when the solar wind speed is less than $425 \mathrm{~km} \mathrm{~s}^{-1}$ to observe near $1 \mathrm{AU}$ no evidence of a strahl population in either the electron solar wind or within the foreshock. Estimating the fluid flow within each energy step returned from the Plasma Electron And Current Experiment (PEACE) on board Cluster-2 often finds that in slow wind the GSE spherical flow angles in energies above where there is a clear core/halo signature are often close to radial with no evidence of a field-aligned flow. This signifies the lack of a strahl presence in the electron velocity distribution function (eVDF). When there is no obvious strahl signature in the data, the electrons above the core/halo in energy appear to be unstructured and smeared in angle. This can either be interpreted as due to statistical noise in low counting rate situations or the result of intense scattering. Regions where the strahl is seen and not seen are often separated by a very thin boundary layer. These transitions in the spacecraft frame of reference can be quite rapid, generally occurring within one to two spins (4-8 s).
\end{abstract}

Keywords. Interplanetary physics (solar wind plasma)

\section{Introduction}

The strahl (Rosenbauer et al., 1976, 1977) represents one of the four populations that make up the electron solar wind. Unlike the thermal, isotropic core, the suprathermal halo (Feldman et al., 1975) and the high-energy super-halo (Lin, 1998; Wang et al., 2012), all of which follow a radial trajectory away from the sun, the strahl flows parallel or antiparallel to the ambient solar wind magnetic field, i.e., it is field-aligned. The general consensus is that the solar wind originates in the solar corona through a mixture of Coulomb scattering and interactions with broadband turbulence (Vocks et al., 2008; Vocks, 2012; Pavan et al., 2013; Che and Goldstein, 2014; Che et al., 2014); however, there is still no clear consensus on the specific mechanisms involved in the formation of any of its four individual populations.

The strahl is thought to be formed from the initial highenergy electron tail in the absence of Coulomb collisions and is focused along the strong magnetic field (Smith et al., 2012; Che and Goldstein, 2014) although recently Seough et al. (2015) have put forward the possibility that the strahl is formed directly from the halo by pitch angle scattering off of whistler waves. In the absence of scattering, the mirror force will focus the strahl in pitch angle as it propagates away from the sun. This strong focusing is, however, not observed. Observations show that the strahl actually broadens with radial distance (Pilipp et al., 1987a, b; Hammond et al., 1996), beginning about where scattering would be expected to dominate over focusing (0.5 AU) (Owens et al., 2008). Several suggestions as to the free-energy sources available to drive the broadening have been put forth (e.g., see Dum et al., 1980; Saito and Gary, 2007a, b; Gary and Saito, 2007; Gary et al., 2008; Viñas et al., 2010). The possible mechanisms include scattering of strahl electrons through interactions with sunward propagating whistler waves (e.g, see Vocks et al., 2008), broadband whistler turbulence (Pierrard et al., 2011) and Langmuir waves (Pavan et al., 2013).

Maksimovic et al. (2005) and Stverák et al. (2009) have shown that the strahl and halo densities vary inversely with radial distance from the sun, implying that at least a portion of the strahl may be degraded in energy and integrated into the halo. The processes active to drive this are not known, but it has been suggested that the same processes responsible for broadening the strahl may also be capable of scattering strahl electrons into the halo. This is envisioned as being a slow and continual erosion of the strahl and a corresponding buildup of the halo through inelastic scattering. 
The characteristics of the strahl in slow wind have been studied by Fitzenreiter et al. (1998), Stverák et al. (2009), and Anderson et al. (2012). They find similar strahl profiles in the fast and slow winds with perhaps a slightly higher density in the fast wind and increased broadening during periods of slow wind. Anderson et al. (2012) in a large statistical study claimed that there was no strahl in about $25 \%$ of the data. This was an automated statistically based study, and analysis is performed only during times when specific criteria are met. They mention no long contiguous time periods of no strahl or any correlation of the absence of the strahl with solar wind speed.

Gurgiolo et al. (2012) have shown observations of what appears to be strong local diffusion of the strahl within the energy range where the strahl and halo overlap, suggesting that the buildup of the halo may occur rapidly within regions where the intense disruption of the lower edge of the strahl occurs. Again, it is not certain what processes are responsible for the observations.

In this paper we will detail a set of initial observations made at $1 \mathrm{AU}$ by the Plasma Electron And Current Experiment (PEACE) instrument on the Cluster spacecraft. The observations show that during time periods of slow wind $\left(\lesssim 425 \mathrm{~km} \mathrm{~s}^{-1}\right)$, the strahl is often not seen. In place of the strahl, there is an unstructured electron plasma that is smeared in azimuth and elevation angle. This unstructured distribution may be a consequence of counting statistics due to low count rates and is possibly coupled with intense scattering.

\section{Data}

This study makes use of data from a number of experiments on board the Cluster spacecraft, including PEACE (Johnstone et al., 1997; Fazakerley et al., 2010), the Fluxgate Magnetometer (FGM) (Balogh et al., 1997; Gloag et al., 2010), the Electric Field and Waves (EFW) experiment (Gustafsson et al., 1997; Khotyaintsev et al., 2010), the Waves of High frequency and Sounder for Probing of Electron density by Relaxation (WHISPER) (Décréau et al., 1997; Trotignon et al., 2010), and the Spatio-Temporal Analysis of Field Fluctuations (STAFF) experiment (Cornilleau-Wehrlin et al., 1997, 2010).

The primary data used in the study come from PEACE. PEACE consists of two hemispherical electrostatic analyzers designated HEEA (high-energy electrostatic analyzer) and LEEA (low-energy electrostatic analyzer). These are separated by $180^{\circ}$ on the satellite and differ only in their geometric factors (HEEA's geometric factor is larger than LEEA's). Despite their names, both can cover the energy range $0.6 \mathrm{eV}$ to $26 \mathrm{keV}$. The analyzers' fields of view are perpendicular to the spacecraft spin axis, which is about $5^{\circ}$ off GSE $-\mathrm{Z}$, and cover $180^{\circ}$ in elevation in 12 sectors. The full $360^{\circ}$ of azimuth is covered in one rotation of the spacecraft so that a three-dimensional snapshot of the electron distribution is accumulated once per spin $(\sim 4 \mathrm{~s})$.

Because of telemetry restrictions, PEACE generally returns only a subset of the total data collected onboard. Exactly what is returned depends on the instrument mode, which can be separately commanded for each analyzer on each of the four spacecraft. The telemetry rate, as well as the amount of data being returned, determines the time cadence at which full three-dimensional distributions are downloaded. During the time intervals used in this paper, all satellites were operating in burst-mode telemetry and PEACE was returning one 3-D distribution per spin. The data were being returned in 6 or 12 elevations bins with 30 or 60 energy steps and 16 or 32 azimuths, depending on the data allocation available.

PEACE data are used to characterize the electron plasma through both moments and visualization tools that allow various aspects of the electron velocity distribution function (eVDF) morphology in velocity space to be highlighted. The FGM five vector per second data are used to characterize the local magnetic field, while the full-resolution FGM data together with the STAFF waveform data are used to construct magnetic field power spectra. Both the EFW and WHISPER are used in the formation of the electron moments; EFW provides the spacecraft potential used to correct the measured electron energy, and WHISPER provides the flags necessary to filter out times during which the computed moments may be contaminated by perturbations created by WHISPER active sounding.

\section{$3 \phi-\theta$ plots}

$\phi-\theta$ plots are used throughout this paper to illustrate features in the eVDFs. This mode of presentation allows one to show the entire three-dimensional distribution function at a given energy as a two-dimensional projection. The three columns of $\phi-\theta$ plots in Fig. 1 serve as examples of some of the features available in the plot format. Each set of $\phi-\theta$ plots uses data from the same eVDF and shows a contiguous set of PEACE energy shells from 30.1 to $110.3 \mathrm{eV}$. To fully characterize an $\mathrm{EVDF}$ requires one $\phi-\theta$ plot per returned energy step; however, in general any feature of interest can be characterized by a small subset of the returned energy steps. The data are plotted as a function of $\phi$ (the spacecraft spin angle) and $\theta$ (the instrument elevation angle). For Cluster, the instrument frame of reference is offset by about $5^{\circ}$ from the $-\mathrm{Z}$ GSE frame of reference so that the ecliptic plane makes an angle of about $-95^{\circ}$ with respect to the spacecraft spin axis $\left(0^{\circ}\right.$ being parallel to the spin axis). A rotation angle of $0^{\circ}$ is the position at which the instrument aperture lies in the plane containing the sun and the spacecraft $x$ axis. Plots are logarithmically scaled in intensity. Individual plots may be auto-scaled as is the left-hand column of plots in Fig. 1 or assigned a common scaling range as in the center and right- 

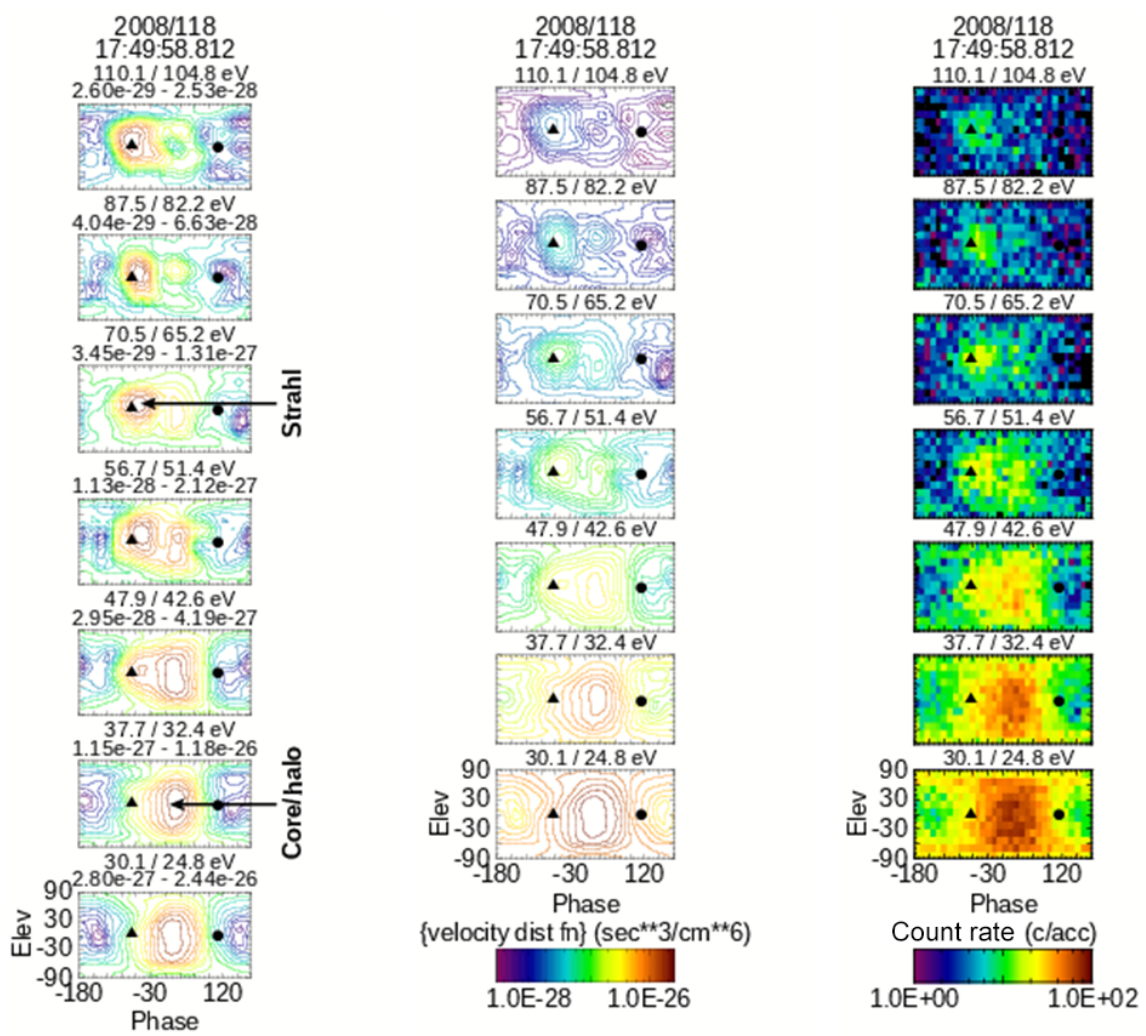

Figure 1. Example $\phi-\theta$ plots of a solar wind eVDF. All columns of plots show the same set of solar wind data but with different formatting options. The first two columns of plots illustrate different scaling formats. The left-hand column of plots is auto-scaled, while the center column uses a common scaling. The strahl and core/halo populations are identified in the first column of plots. The right-hand column of plots shows the data in raw format $\left(\mathrm{cnts} \mathrm{acc}^{-1}\right)$ allowing for the statistical importance of the data to be followed. Individual colored tiles are identical in area to the instrument angular resolution.

hand column of plots. When auto-scaling is used, the range is shown above each plot, while when using a common scaling range the scaling is given in a color bar at the bottom of the column. The energy associated with each individual plot is also shown above it in the following format: raw center energy-potential corrected center energy. The dot and triangle in the plots are the projections of the average magnetic field head and tail, respectively.

The three columns of $\phi-\theta$ plots in Fig. 1 make use of different display options. The eVDF displayed is typical of that seen in the solar wind and the strahl and core/halo populations are explicitly labeled in the left set of plots. The core and halo both flow radially away from the sun and overlap significantly in phase space, which makes it impossible to separate the two populations in a $\phi-\theta$ plot. For this reason we refer to the two populations within a $\phi-\theta$ plot by the single name core/halo. The strahl is field-aligned and approximately centered on one of the magnetic field projection points depending on whether the flow is parallel or antiparallel to $\boldsymbol{B}$. When the magnetic field has an large off-radial component, the strahl will be shifted off the core/halo as it is in Fig. 1. Even with a small off-radial component, the shift in the strahl will be evident although there still may be a considerable overlap of the populations. The first and last two columns of plots in the figure illustrate different scaling formats. The left-hand column of plots is auto-scaled, while the center and right-hand columns use a common scaling for all plots. The right-hand column of plots shows the data plotted in units of counts per accumulation (cnts acc ${ }^{-1}$ ) with all smoothing and contouring turned off. Here each grid cell in the plot exactly matches the instrument angular resolution.

\section{Observations}

The basic solar wind or foreshock eVDF can tentatively be broken in energy such that below the break point the core/halo form the dominant electron population and above the break point the strahl (and possibly any return foreshock electrons) are the dominant population(s). The energy range above the break point will be defined as the nominal strahl energy range in this paper. In Fig. 1 the break point occurs at about $70.5 \mathrm{eV}$. It is common to find that when the solar wind speed is less than $425 \mathrm{~km} \mathrm{~s}^{-1}$, there is no strahl population observed above the break point and that any electrons 


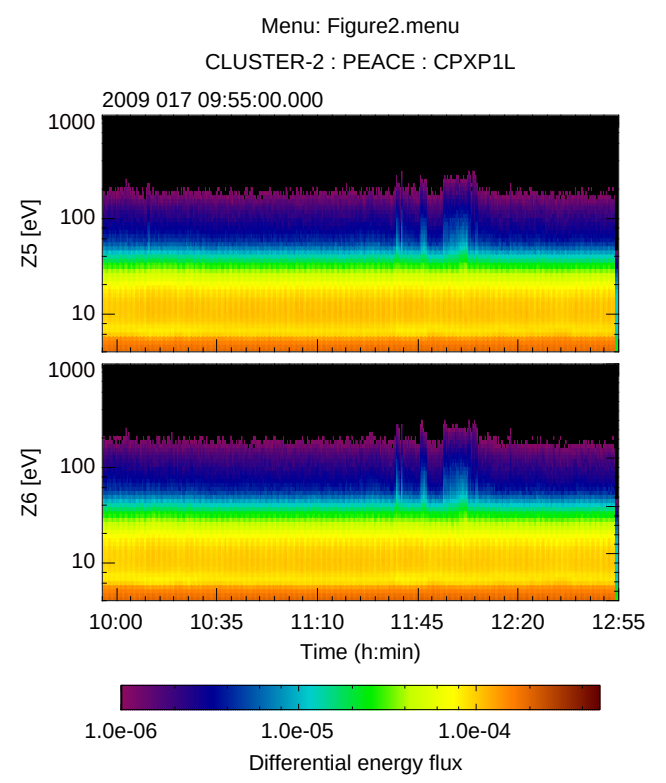

Figure 2. Spectrograms of the PEACE elevation zones above and below the ecliptic plane. This is basically solar wind with some foreshock. Bursts of intense high-energy electrons above $40 \mathrm{eV}$ correspond to periods when the spacecraft are in the foreshock. The bursts occur due to the addition of higher-energy return electrons that have been backscattered off of, or leaked through, the shock.

located above this energy appear to be disorganized and diffusely spread in angle. The disorganization is most directly seen in the $\phi-\theta$ plots but can also be inferred through plots of the GSE spherical electron fluid velocity angles determined at each returned energy step.

We have identified 37 intervals from the 181 we analyzed that have no observable strahl. In this paper we will look in depth at two such periods, presenting the basic characteristics observed in the plasma and contrasting them to what is observed in typical solar wind. The features illustrated in the two events are common to one degree or another in all 37 events where no strahl was observed. The first interval spans the time from 09:55 to 12:55 UT on 17 January 2009 and the second that from 22:15 to 23:43 on 28 January 2011. During both events PEACE was returning data in 60 energy $\times 16$ azimuth $\times 6$ elevation bins. We used data from only a single spacecraft in analyzing each event. Generally this was $\mathrm{C} 2$ unless it was not returning data, in which case data from $\mathrm{C} 3$ or C4 were used. In $2011 \mathrm{C} 2$ lost one of its extreme elevation sensors, and thereafter the data used came from either $\mathrm{C} 3$ or C4.

\subsection{The 17 January 2009 09:55 to 12:55 UT event}

Figures 2 and 3 characterize the electron plasma observed during the event. The populations centered on the magnetic head are moving sunward and those centered on the magnetic tail are moving anti-sunward. As such, the strahl (when

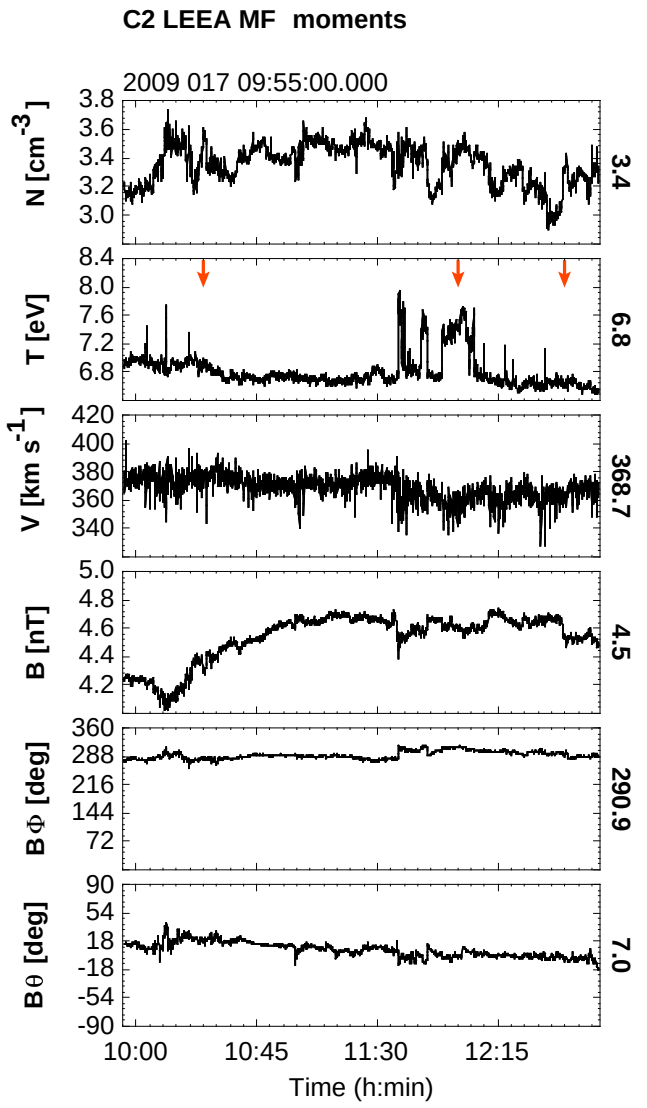

Figure 3. Plots of the total electron density, temperature, and speed and magnetic field components in spherical coordinates across the time interval in Fig. 2. The enhancements in the total temperature between 11:30 and 12:15 indicate times when the spacecraft was in the foreshock.

present) will be centered on the magnetic tail (triangle) and the return electrons (when the spacecraft are in the foreshock) will be centered on the magnetic head (solid circle) in the $\phi-\theta$ plots.

The dual spectrograms in Fig. 2 show the general electron characteristics during the event. The data are taken from the two LEEA elevations that bracket the ecliptic plane. This period is almost entirely comprised of solar wind with a few short transitions into and out of the foreshock. The foreshock transitions are identified by intensification in the electron spectrum above about $40 \mathrm{eV}$ (viz., between 11:35 and 12:05 UT). The intensifications are the result of the addition of higher-energy return electrons to the spectra. (As will be shown, there is no observed strahl during this event.) Figure 3 shows the electron moments and magnetic field during the event. From top to bottom are the total electron density, temperature, fluid speed, and the spherical components of the magnetic field. All vector quantities are in GSE. To the right of each plot are the average values of the quantities plotted. The average local solar wind speed across the event is $368.7 \mathrm{~km} \mathrm{~s}^{-1}$, which can be contrasted with the average so- 

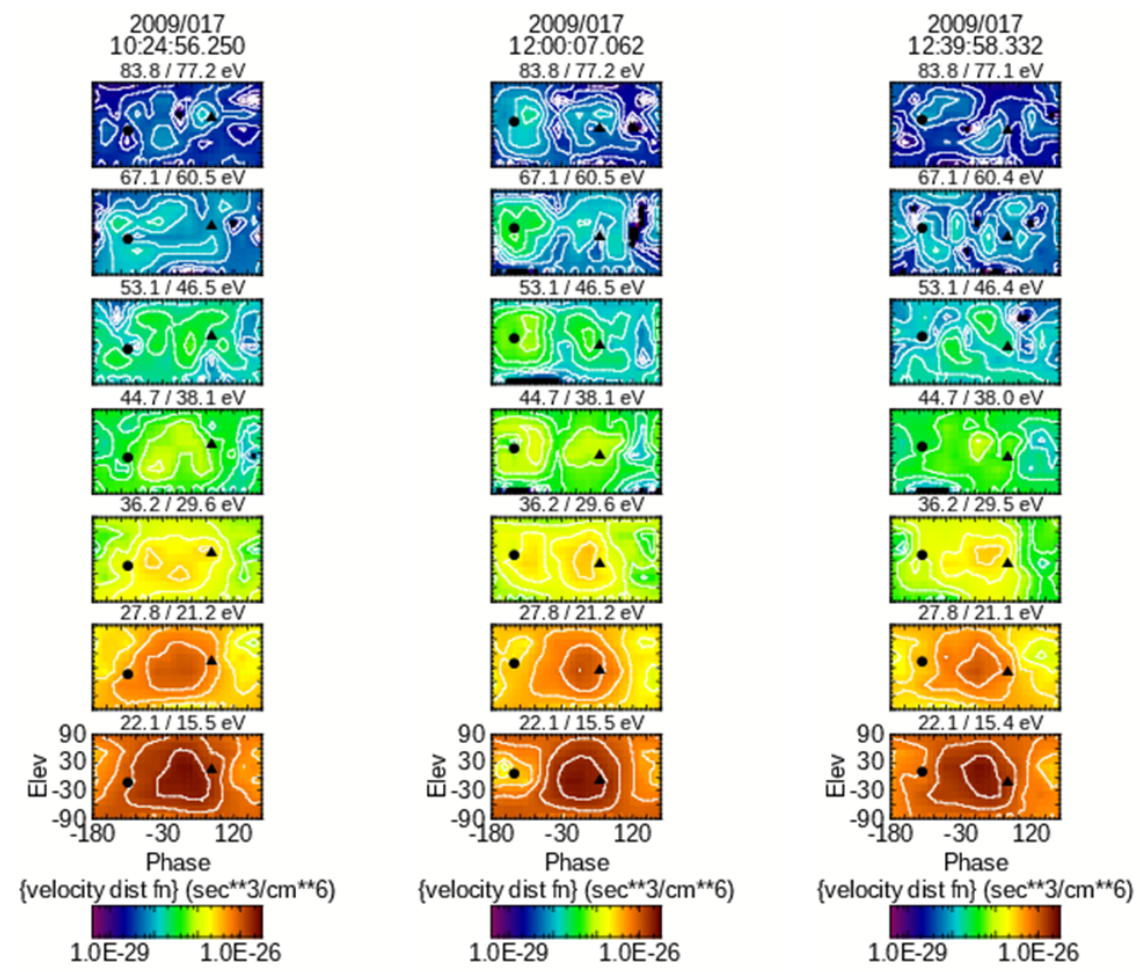

Figure 4. Three sets of $\phi-\theta$ plots from the event in Figs. 2 and 3. Red arrows in Fig. 3 show the locations of the data in each column of plots. The center column of plots is from a foreshock interval as evidenced by the presence of a return electron population.

lar wind speed of $355.7 \mathrm{~km} \mathrm{~s}^{-1}$ measured by the Advanced Composition Explorer (ACE) at L1. The excursions into the foreshock occur in conjunction with increases in the electron temperature.

Figure 4 shows three columns of $\phi-\theta$ plots which illustrate the typical characteristics of the eVDF across the event in Figs. 2 and 3. The red arrows in Fig. 3 show the locations where the eVDFs were taken. From left to right this corresponds to the left to right columns in Fig. 4. The event basically consists of two long stretches of solar wind separated near 12:00 UT by several excursions into and out of the foreshock. The first and last columns of plots in the figure are taken from within the two solar wind intervals, while the center column is taken from within the foreshock. Each column of plots depicts every other returned energy step between 22.1 and $83.8 \mathrm{eV}$, inclusively. A clear core/halo population is seen in each column up to about $44.7 \mathrm{eV}$. There is also a return electron population in the middle column of plots that is centered on the head of the magnetic field projection and can be seen in all the energy steps shown. In none of the three eVDFs, however, is there any evidence of a strahl population. Above $44.7 \mathrm{eV}$ the electrons appear to be unstructured (except in the foreshock, where the return population is clearly evident).

One of the primary concerns with the observations during periods where the plasma appears to be unstructured is whether this is the result of low counting rates and therefore statistical in nature or if the small-scale variations in the data are significant and created by some physical process such as scattering. Figure 5 shows the same sets of $\phi-\theta$ plots as shown in Fig. 4, with the exception that the units plotted are $\mathrm{cnts} \mathrm{acc}^{-1}$. These plots are individually auto-scaled, which makes the maximum scaling (first line above each plot) the highest number of counts in any cell in the plot grid. The value to the right of each plot is the average counts per cell in the plot grid (total number of counts in the plot grid divided by the total number of cells). Colors vary similarly to the color bar shown in Fig. 2 but run from the maximum to minimum scaling value for each plot. Once above the energy range where the core/halo can be easily observed $(44.1 \mathrm{eV})$, the maximum cnts acc ${ }^{-1}$ falls off rapidly and at the upper energy step included in Fig. 5 is down to $7 \mathrm{cnts} \mathrm{acc}^{-1}$. At this level statistical variations become a concern. However, statistical variations are really only important if there is no long-term mean in the data, that is the variations represent pure noise and not a scatter about a non-zero mean. In the latter there is at least some degree of organization present, suggesting that the data are not reflecting pure noise.

There are two methods to look for noise within the data. The first of these is shown Fig. 6. This figure contains two sets of plots with identical formats except that the upper set covers the full event time, while the lower set covers the lim- 

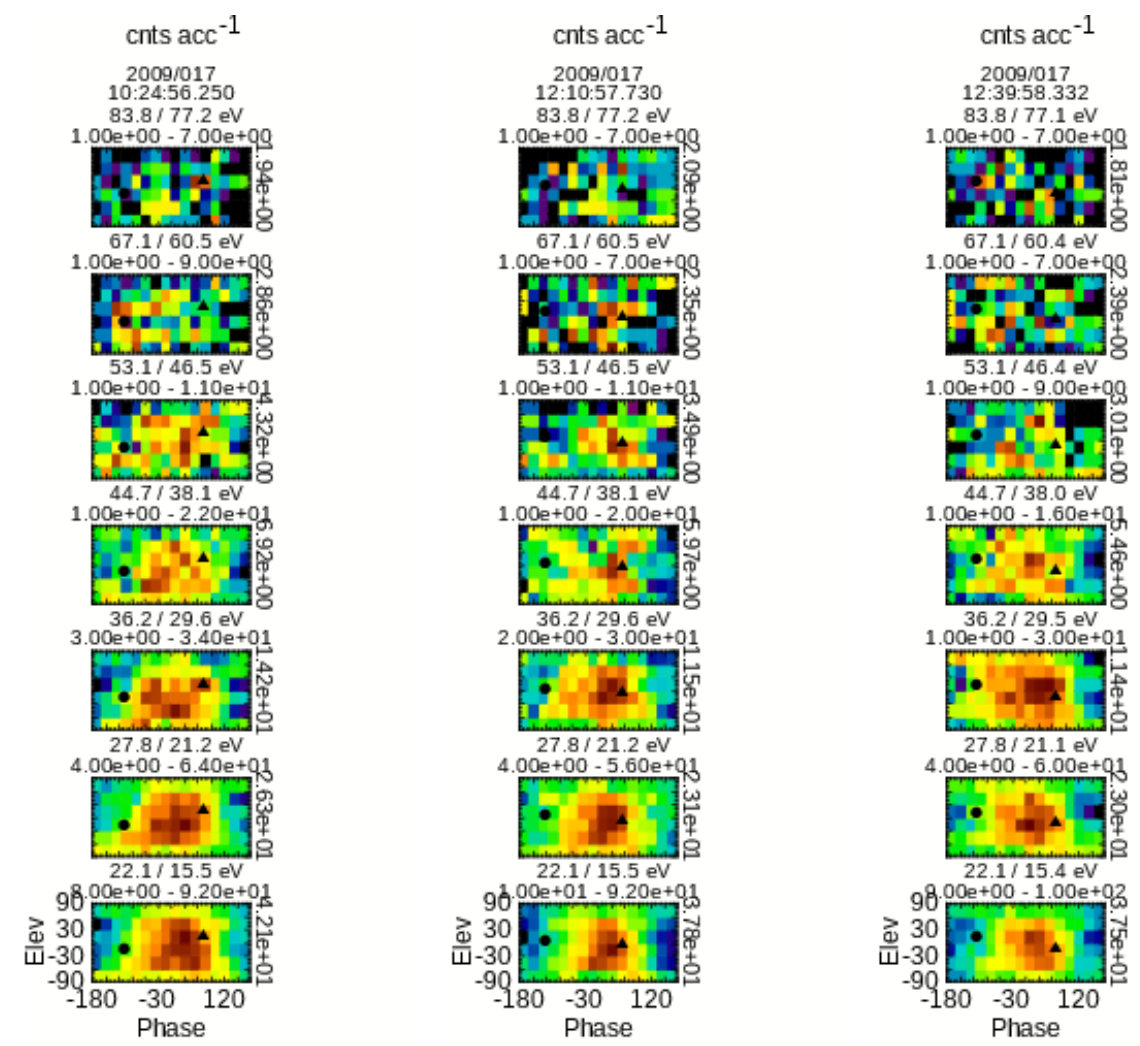

Figure 5. $\phi-\theta$ plots in units of $\mathrm{cnts}_{\text {acc }}{ }^{-1}$. Each column of plots corresponds to the column of plots in Fig. 4. The plots are auto-scaled and the maximum cnt/acc scaling above each plot provides the highest count level. The value to the right of each plot is the average counts per cell in the data grid.

ited time period 11:20 to 12:20 UT, which includes the foreshock excursions. The upper plot in each set shows the GSE azimuthal flow angle as a function of time computed in each of 12 included energy steps. The energy step traces are colorcoded according to the list of energies at the right of the top plot in each set. The lower plot in each set shows the GSE elevation flow angle as a function of time. The thick black and yellow traces are the head and tail azimuth and elevation angles of the magnetic field vector, respectively. In this format energies that are primarily made up of core/halo particles are expected to show radial flow $(\phi=0, \theta=0)$, while energies which are predominantly strahl and/or return particles are expected to be field-aligned (i.e., following either the black or yellow curves). Nominally, energies at which the eVDF is transitioning from radial to field-aligned flow should, with increasing energy, show the flow gradually changing from radial to field-aligned.

Energies of $27 \mathrm{eV}$ and below in Fig. 6 basically exhibit a radial flow across the event. At these energies the dominant population is the core/halo (see Fig. 4). Above $27 \mathrm{eV}$ the flow is still basically radial at all energies except when the spacecraft is in the foreshock. The lower set of plots in Fig. 6 covers the time period when the spacecraft are transitioning between the solar wind and foreshock. In the foreshock above
$27 \mathrm{eV}$, the flow progressively changes from a predominantly radial flow until at $67 \mathrm{eV}$ and above it primarily a sunward, field-aligned flow (becoming aligned with the black trace). Change occurs as the intensity of the core/halo rolls off with energy in the presence of the foreshock return electrons. Had there been a strahl population the curves would undoubtedly have shifted towards the yellow trace since the strahl is generally the more dominant of the two populations and would have moved opposite to the return population.

The fact that at times the high-energy electrons show an almost radial flow is indicative of the fact that there is neither a strahl nor a return population present in the eVDF. The near radial flow is expected if the plasma at those energies is unstructured. It does not, however, convey any information as to whether the lack of structure is simply a result of low count rates and counting statistics or has a physical origin.

With increasing energy there is an increase in the statistical variance in the traces, especially at the upper two energy steps. This increase in the variance is a result of the decrease in $\mathrm{cnts} \mathrm{acc}^{-1}$. Note that the variance has a wider spread in the solar wind than it does in the foreshock where return electrons tend to dominate the computed flow angles suppressing much of the variation. In the solar wind, what is seen in the upper two energy channels is probably statistical noise. 

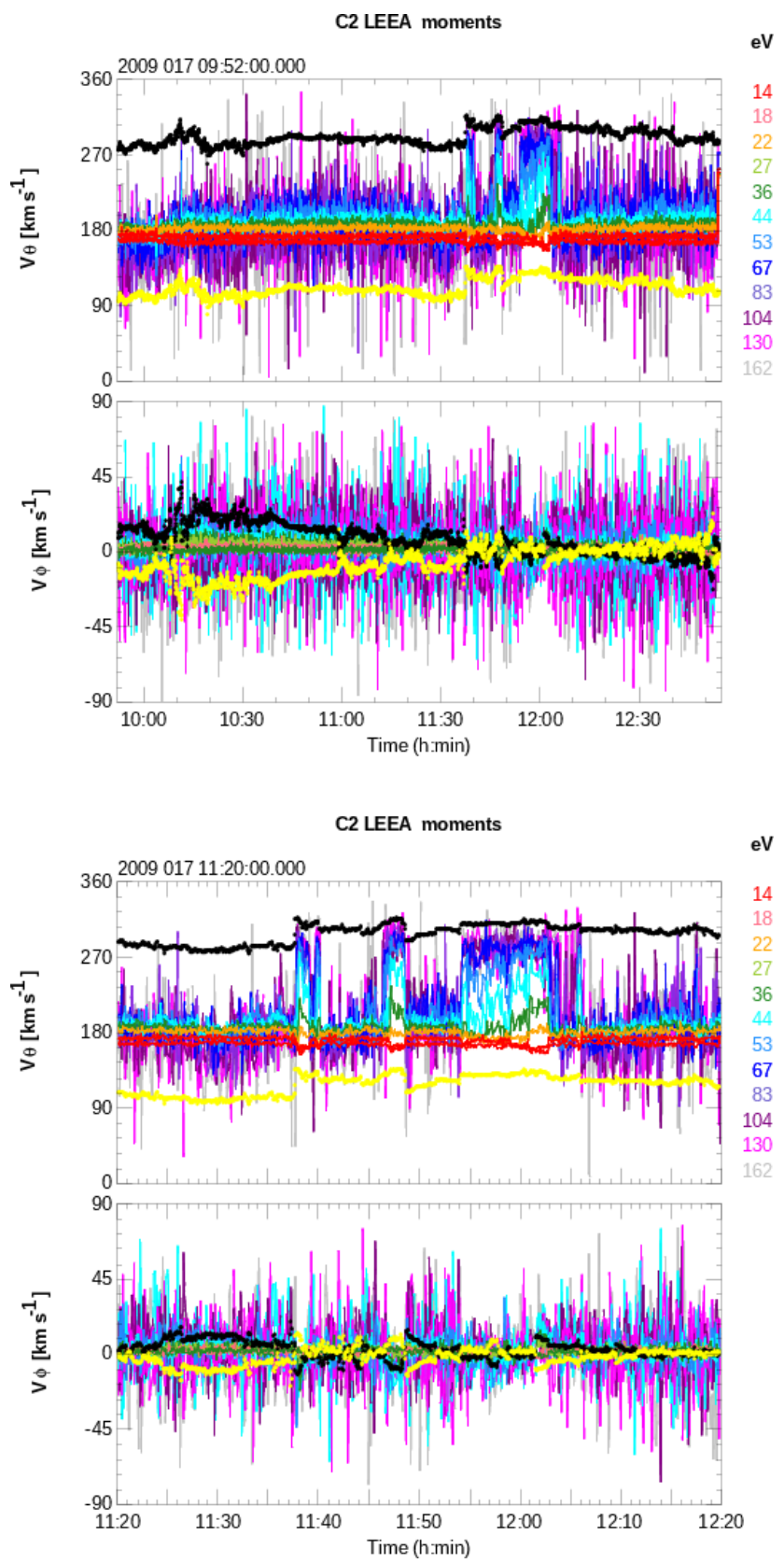

Figure 6. Upper plot shows the electron fluid flow GSE angles at 12 different energies over the full event time. Black and yellow lines are the spherical angles associated with the head and tail of the magnetic field vector. The lower plot shows a portion of the upper plot at higher time resolution.

There is another test that can be made to indicate the relevance of the data within a plot grid at a single energy step. This is done by assuming that the data within each grid cell in a plot follow a Poisson distribution function. The Poisson distribution expresses the probability that a given number of events will occur within a fixed time interval as

$$
P(x)=\frac{\lambda^{x} e^{-\lambda}}{x !},
$$

where $x$ is the number of events in the fixed interval and $\lambda$ is the average number of events per interval (rate of occurrence). The probability in any given grid cell is based solely on the average count rate in that cell within a fixed interval. We use an interval that is one accumulation period, the length of which depends on how the data are averaged together prior to transmission. The rate of occurrence in an interval is assumed to be constant and all events are assumed to be independent. We estimate $\lambda$ within each grid cell in a $\phi-\theta$ plot from the average of 25 successive $\phi-\theta$ plots. Each grid cell is considered as an independent system, which is necessary to allow for any angular dependence in the plot (which depends on the angular dependence of the measured electron distribution). A mapping of the Poisson distribution from a $\phi-\theta$ plot grid is made by setting the measured count rate in each cell to $x$ in Eq. (1) and $\lambda$ to the average count rate observed in the cell and then estimating $P$, which is the expected probability of observing $x$ counts in any given accumulation period. Given $P$, we would expect to see $x$ counts in the cell once every $1 / P$ accumulation periods; the smaller $P$, the less likely that the observed count rate is simply statistical in nature.

Figure 7 shows three columns of $\phi-\theta$ Poisson-based statistical maps corresponding to the top three plots in each column in Fig. 5. The colors translate into the Poisson probability according to the color bars at the bottom of each plot column. Basically colors from brown down through green denote pixels where the variations in the data are reasonably consistent with simple statistical variations and the blue through purple pixels denote regions where the count rates are more likely to be the result of some physical process. Even where the variations in the data are consistent with statistical noise, the underlying data may still be physical. We know that at least in the second column there is a return electron population and although the variations seen in this population may be purely statistical, the underlying data are real. The Poisson analysis suggests that not all of the unstructured spread observed in the electrons results from statistical noise - there are a fair amount of counts that appear to be physical in origin.

\subsection{The 28 January $201122: 15$ to 23:43 event}

This is a slightly more dynamic and complex interval than that discussed in Sect. 4.1. We use figures similar in context and format to those used to describe the 17 January 2009 event, and except where necessary, we will not describe the formats. Figures 8 and 9 characterize the electron plasma over the event time. As in the previous event, populations centered on the magnetic head are moving sunward and those centered on the magnetic tail are moving anti-sunward. Figure 8 shows spectrograms from two LEEA elevations, one 

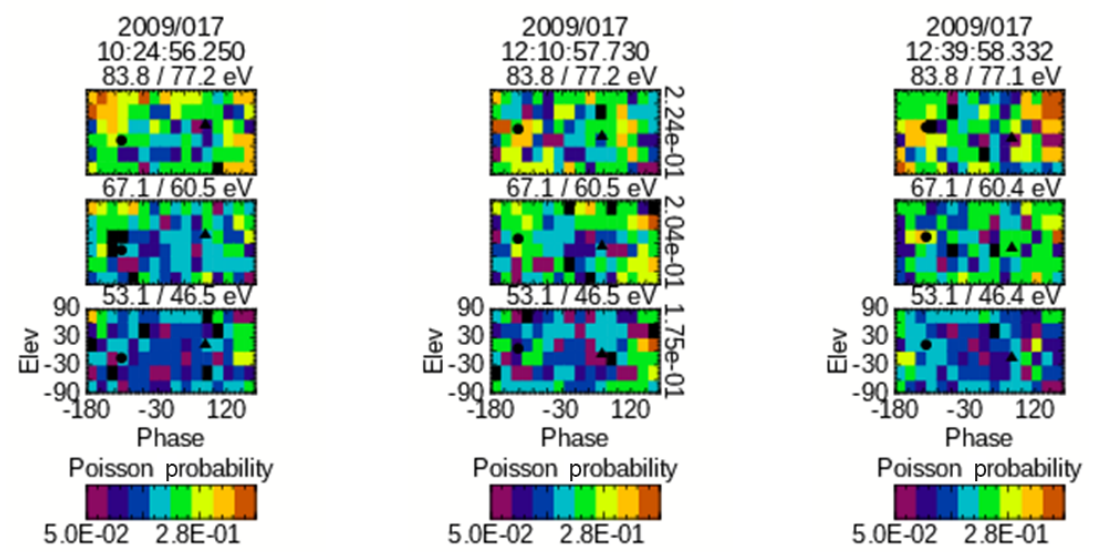

Figure 7. $\phi-\theta$ plots of the Poisson statistics associated with the top three energy steps in each of the three columns in Fig. 5.

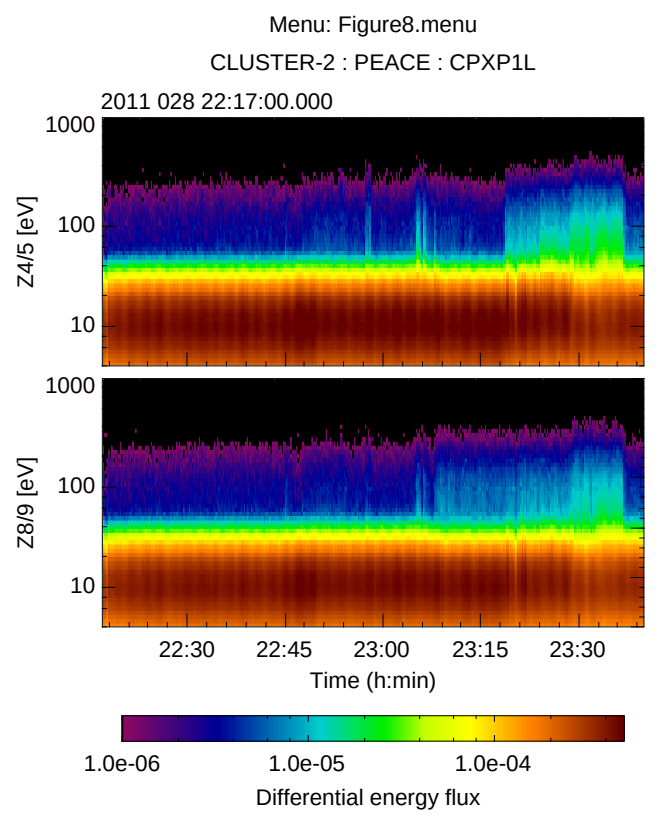

Figure 8. Spectrograms of the PEACE elevation zones above and below the ecliptic plane from the second event. Enhancements in the electron intensity above $60 \mathrm{eV}$ are times when the spacecraft are in the foreshock. The enhancements are due to return electron populations from the foreshock and also times when the strahl is present.

viewing just below the ecliptic plane and one viewing about $45^{\circ}$ above the ecliptic plane. These elevations ensure that both projections of the magnetic field are in the field of view. Enhancements in the greater than $40 \mathrm{eV}$ electron intensity in the upper spectrogram are mainly due to the strahl, while similar enhancements in the lower spectrogram are mainly due to return electrons. The different response of the sensors is due to the orientation of the magnetic field and which sensor is looking parallel and which antiparallel to field. Up to
C2 LEEA MF moments

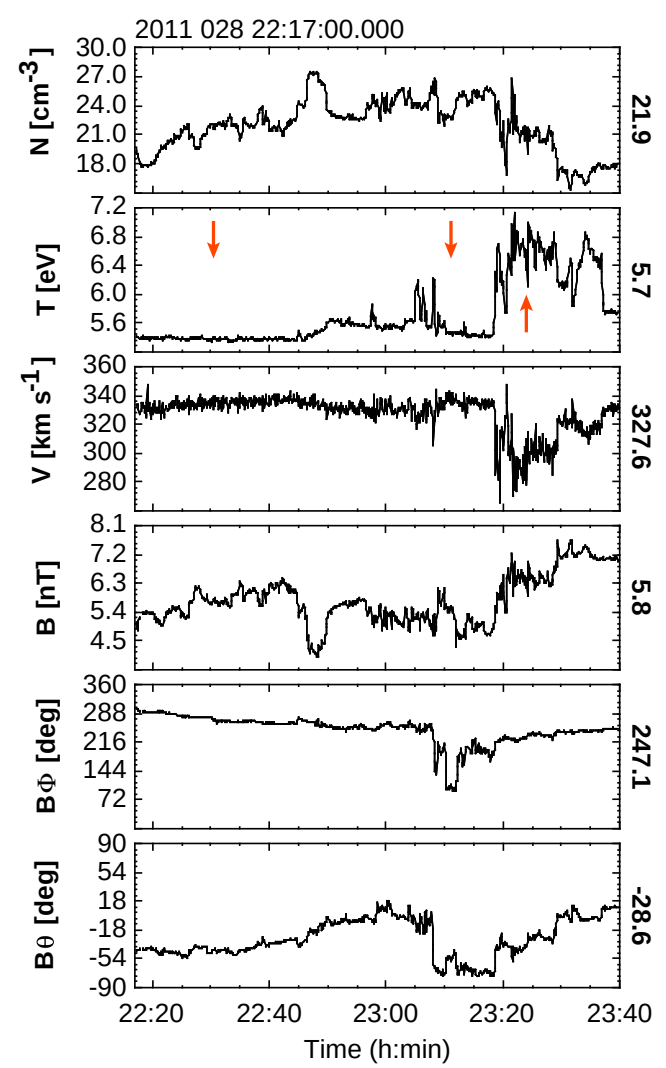

Figure 9. Plots of the total electron density, temperature, and speed and magnetic field components in spherical coordinates across the time interval in Fig. 8. The enhancements in the total temperature between 23:20 and 23:35 indicate times when the spacecraft was in the foreshock. Red arrows in the figure show the locations of the data in the columns shown in Fig. 10. 

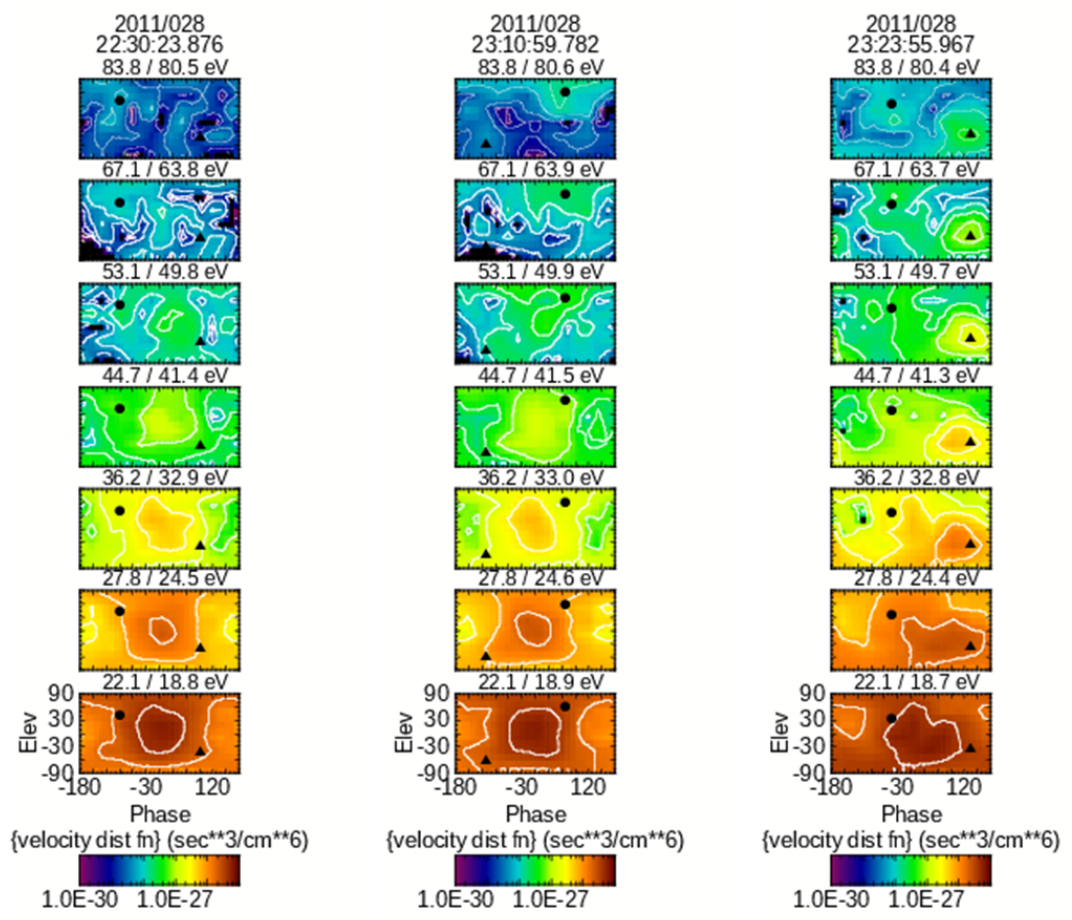

Figure 10. Three sets of $\phi-\theta$ plots from the event in Figs. 8 and 9. Red arrows in Fig. 9 show the locations of the data in each column of plots. The last two columns of plots are from foreshock intervals. The first shows no strahl, but both have return electron populations.
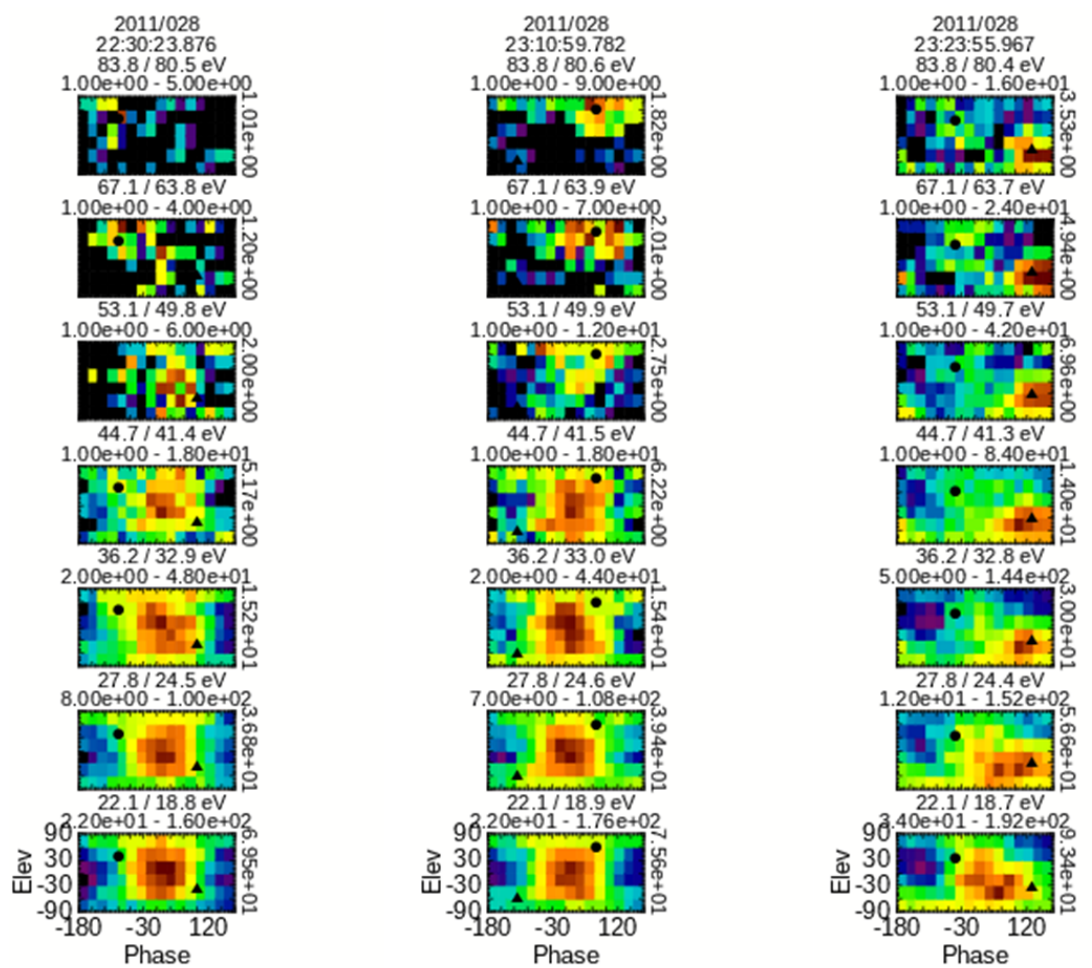

Figure 11. $\phi-\theta$ plots in units of cnts acc ${ }^{-1}$. Each column of plots corresponds to the column of plots in Fig. 10. The plots are auto-scaled and the maximum cnt/acc scaling above each plot provides the highest count level. The value to the right of each plot is the average counts per cell in the data grid. 
about 22:48 UT there is no strahl and after that the strahl is only sporadically present until $23: 18$ to $23: 37$ when a strong strahl signature reappears.

Figure 9 shows the plasma moments and the magnetic field vector in spherical coordinates covering the second event. As in the first event, most of the defining features are seen in the electron temperature. The temperature runs through several different levels of intensity across the event. From the start the temperature takes on a baseline value of about $5.4 \mathrm{eV}$ and later jumps to about $5.6 \mathrm{eV}$ at 22:45 UT. There is no strahl in the data until at least 23:05 UT when the temperature makes narrow jumps to about $6.0 \mathrm{eV}$. These are due to appearance of a strahl that can be seen in the $\phi-\theta$ plots (not shown). Between 23:10 and 23:18 there is no consistent evidence of a strahl, but there are return particles. At 23:18 a large and dynamic increase in the temperature begins that lasts until 23:37 UT. This is a clear foreshock interval with both return and strahl electrons present in the data. During this time the solar wind speed shows good anticorrelation with the temperature. Both the temperature and solar wind speed are modulated by changes in the intensity and the dynamical mixing of strahl and return populations. At 23:37 the temperature returns to a lower value and remains there until the end of the burst-mode telemetry period. The lower temperature coincides with the disappearance of the strahl.

The average local solar wind speed for the event is $327.6 \mathrm{~km} \mathrm{~s}^{-1}$, close to the average speed at ACE, which was $305.2 \mathrm{~km} \mathrm{~s}^{-1}$. This is one of the lowest average speeds that we have seen for any event in this study.

Figure 10 shows three columns of $\phi-\theta$ plots, which illustrate the characteristics of the eVDFs at different times through the event. The red arrows in Fig. 9 show the times of the eVDFs. Left to right corresponds to the left to right columns in Fig. 10. There is no strahl in either of the first two columns of plots, but it is clearly seen in the last column of plots. The last two columns contain a return population, indicating that at these times, the spacecraft is in the foreshock. These same sets of plots are shown in Fig. 11 where the data are plotted in units of $\mathrm{cnts} \mathrm{acc}^{-1}$. All the plots are auto-scaled and show a progressive increase in the maximum counts contained in the plot grid across the columns in the top three plots. The 4 and 5 count maximums in the top two plots in the first column increase to 16-24 counts by the third column, by which point statistical noise is unimportant.

Figure 12 shows the GSE spherical fluid flow angles computed at every other energy step from 14.3 to $162.6 \mathrm{eV}$, inclusive. The upper plot covers the entirety of the event while the lower plot shows just the second half of the event where the plasma begins to show a strong tendency toward fieldaligned flows at the higher energies. The flows in the energies below $53 \mathrm{eV}$ are primarily radial across the entire event. There is no indication of a field-aligned flow until about 22:48 UT, which coincides with the first jump in temperature. This is seen in the energy steps from $67 \mathrm{eV}$ and higher and is a sunward flow consistent with what would be gen-

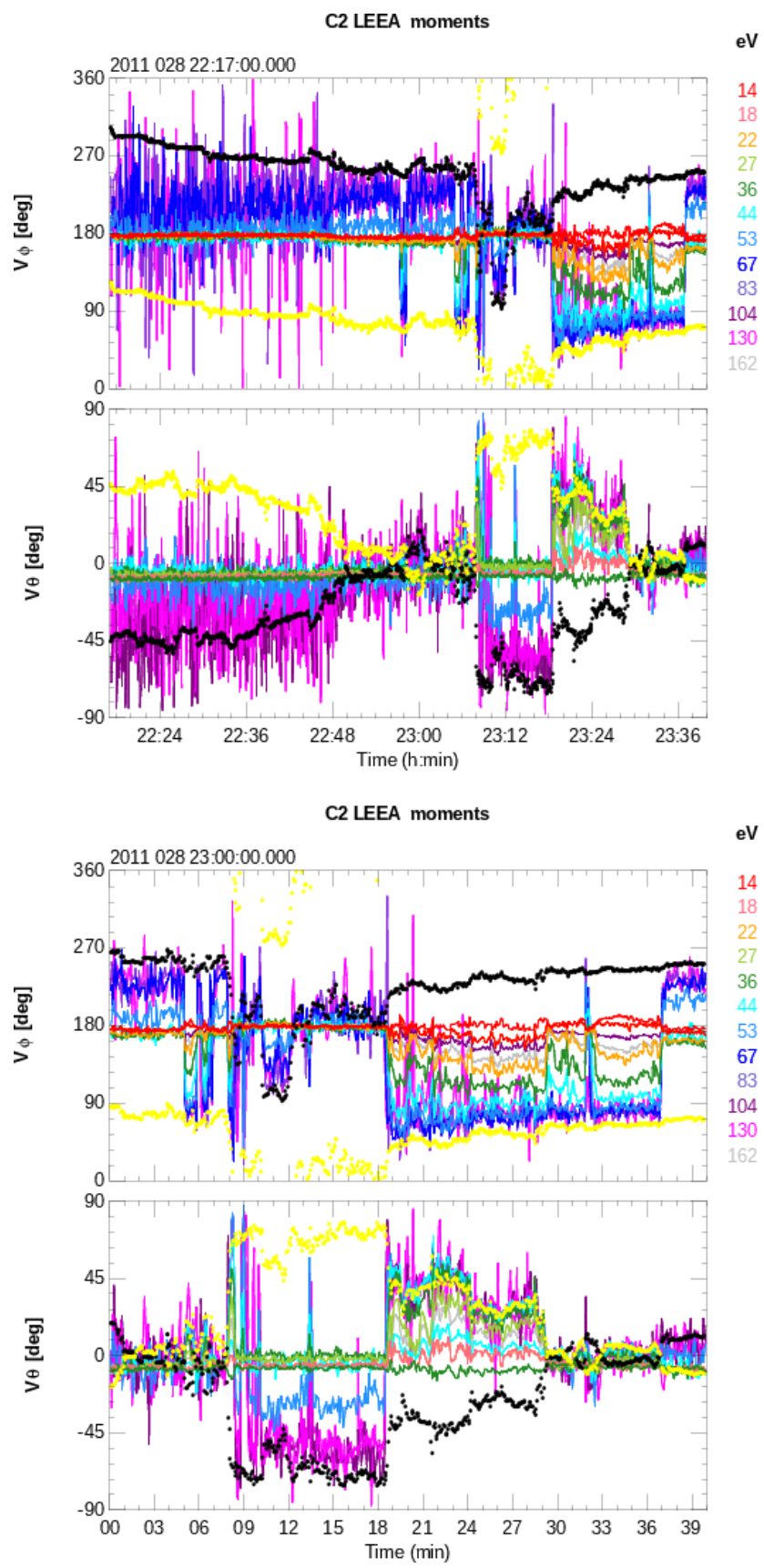

Figure 12. Upper plot shows the electron flow direction in GSE at 12 different energies over the full event time. Black and yellow lines are the spherical angles associated with the head and tail of the magnetic field. The lower plot shows a portion of the upper plot at a higher temporal resolution.

erated by the return electrons. There are two flow reversals between 23:05 and 22:08 UT to an anti-sunward flow (clear in the lower two plots) occurring in conjunction with observations of the strahl in $\phi-\theta$ plots (not shown). By 23:09 UT the field-aligned flow again becomes sunward and stays such until 23:18 UT where it again switches to anti-sunward flow 

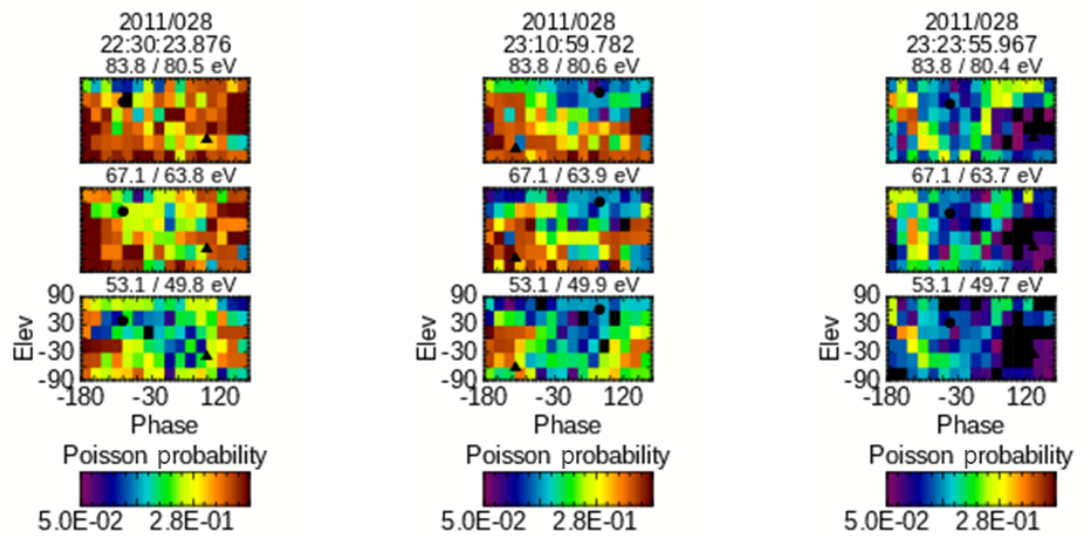

Figure 13. $\phi-\theta$ plots of the Poisson statistics associated with the top three energy steps in each of the three columns in Fig. 11 .

due to the appearance of the strahl in the eVDF (see third column of plots in Fig. 10). Near the end of the event there is final switch back to sunward flow.

Figure 13 shows three columns of $\phi-\theta$ Poisson maps corresponding to the top three plots in each column in Fig. 11. In the top two plots in the first column the data appear to be consistent with statistical noise, probably arising from the low count rates at this time. In the last two columns any variations associated with return and/or strahl populations appear to be well accounted for by a mixture of statistical and nonstatistical variations. Variations in the return and strahl populations in the last two columns of plots would seem to be physical in nature (fluctuations in the source intensity) and not statistical.

\section{Discussion}

We have looked at every interval between 2001 and 2012, inclusive, when the spacecraft are both upstream of the bow shock and returning data using burst-mode telemetry. There are 181 such intervals, of which 37 contained times when the strahl was absent. The absence of the strahl appears to have some dependence on the solar wind speed, occurring primarily during periods of slow wind $\left(\lesssim 425 \mathrm{~km} \mathrm{~s}^{-1}\right)$. The probability of occurrence appears to increase with decreasing wind speed.

Figure 14 illustrates the difference in the observed solar wind speed and temperature anisotropy during periods when the strahl is present and absent. The top two plots in the figure show probability distribution functions (PDFs) of the solar wind speed and temperature anisotropy during the 37 periods where the strahl is absent, while the bottom two plots show the same for the 144 periods when the strahl is present. The PDFs are normalized to area (number of events in each bin divided by the total number of events in the plot) so that the sum of the PDF values in any plot is 1.0. Averages of the quantities in each plot are shown either next to the right or left

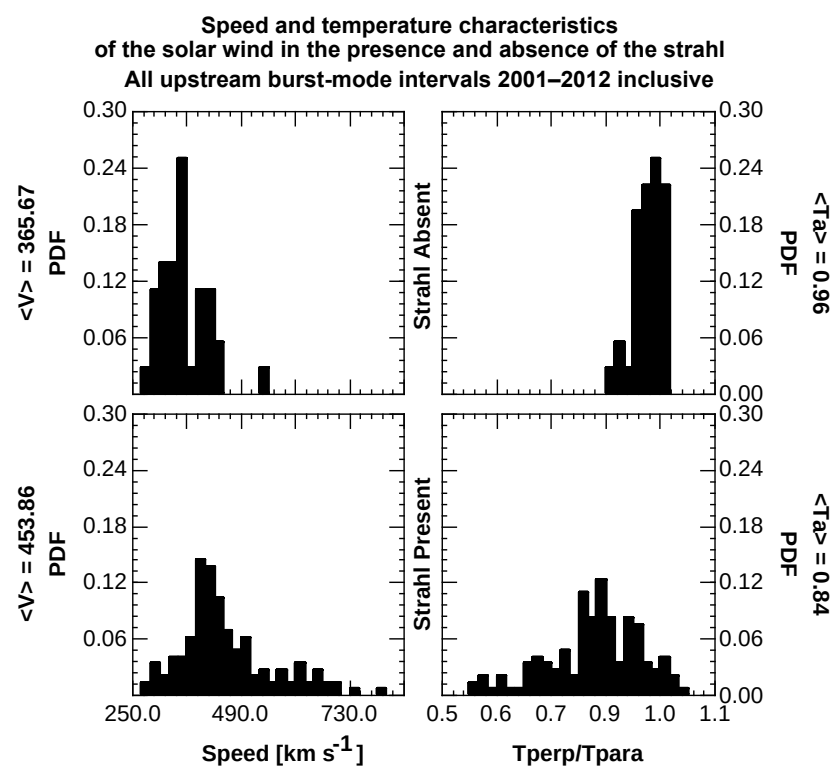

Figure 14. Probability distribution functions of the solar wind speed and total temperature anisotropy during periods when the strahl is absent (top row) and present (bottom row) strahl.

$y$ axis. PDFs of the speed and anisotropy data when the strahl is present are basically what is observed in the majority of pure solar wind events (see, e.g., Newbury et al., 1998). The spread in the temperature anisotropy is a consequence of the presence of the field-aligned strahl, which is often observed to have temperature ratios greater than 1 and as high as 3 (Viñas et al., 2010). Intervals when there is no strahl occur almost exclusively at lower solar wind speeds. Such intervals have a temperature anisotropy close to 1 (the approximate temperature anisotropy associated with the core/halo).

Why the strahl is absent is not clear. The primary reason for the uncertainty is that the current set of observations are not taken in regions where the actual disruption of the strahl 


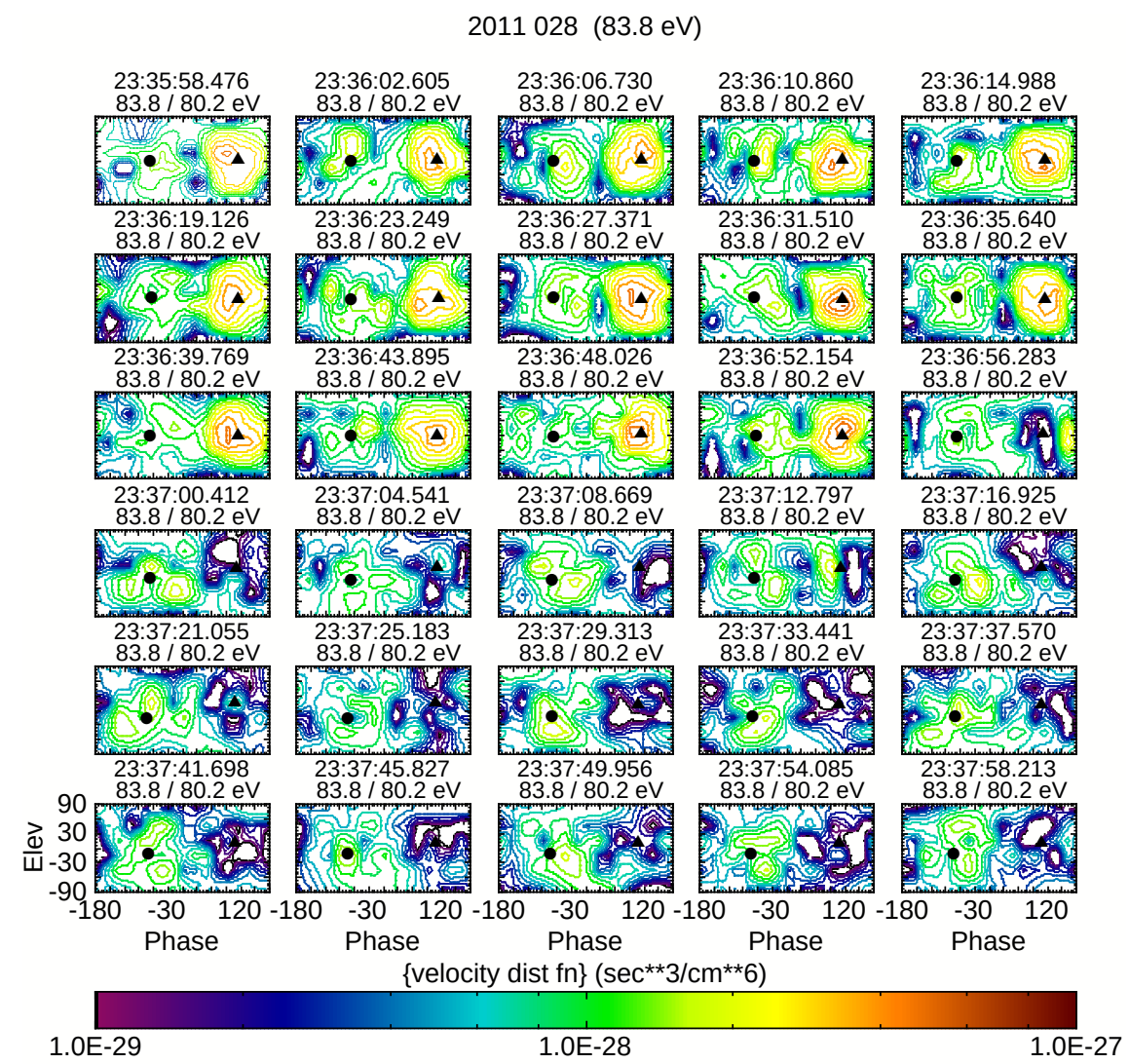

Figure 15. Contiguous temporal set of $\phi-\theta$ plots at $83.8 \mathrm{eV}$ showing the rapid transition from a region with a strahl to one without. Time starts at the top left and runs to the right from top to bottom. The entire set of plots is from within the foreshock.

is taking place. Without knowing where this occurs and under what plasma conditions it occurs, it is almost impossible to begin to speculate on the mechanisms behind it. Given what little information we have, however, two of the more likely explanations are scattering through intense broadband whistler turbulence (Pierrard et al., 2001, 2011) and the possibility that there was no initial formation of the strahl in the inception of the solar wind.

We have looked at the magnetic field spectral power during times when there is no observable strahl, an example of which is shown in Fig. 16. The spectrum is a combination of spectra built from the full-resolution FGM data (blue portion of the total spectrum) and the STAFF waveform data (orange portion of the spectrum), which are normalized at their overlap between 1 and $2 \mathrm{~Hz}$. There is no indication of any local intensification(s) in the power in the STAFF portion of the spectrum, which might indicate the presence of intense local turbulence. If we are using this as a signature of scattering, then this is not locally active or has already taken place farther upstream. It should be noted that foreshock spectra in the presence of a strahl signature do tend to show broadband intensity enhancements in the STAFF portion of the spectrum (between 10 and $100 \mathrm{~Hz}$ in this figure). These enhancements are more than likely evidence of whistler or other broadband turbulence driven by the free energy in the counterstreaming return and strahl electron populations in the foreshock.

The problem with using scattering to fully disrupt the strahl is that it is generally not intense enough and provides no obviously viable means to explain the observations of side-by-side regions of unstructured and structured solar wind eVDFs separated by narrow transition regions. These observations are puzzling, suggesting that other factors are active in addition to solar wind speed. Figures 6 and 12 show that the transition from a plasma with a strahl to one without occurs very rapidly and often without any significant difference in wind speed. A detailed example of one transition is given in Fig. 15 This shows consecutive plots of the $88.3 \mathrm{eV}$ energy step across the last transition from a strahl-dominated to a non-strahl plasma in Fig. 12. Between 23:36:52 and 23:36:56 UT, the strahl drops out of the eVDF with no effect seen in the return population. The existence of the return population across the time period means that there is no transition between the foreshock and solar wind. It is difficult to understand how these boundaries are set up and maintained.

A second possibility is that the strahl is not uniformly produced in the corona with the solar wind. In this scenario the mixture of regions at $1 \mathrm{AU}$ with a presence or absence of the strahl might be the result of the mixing of flux tubes 


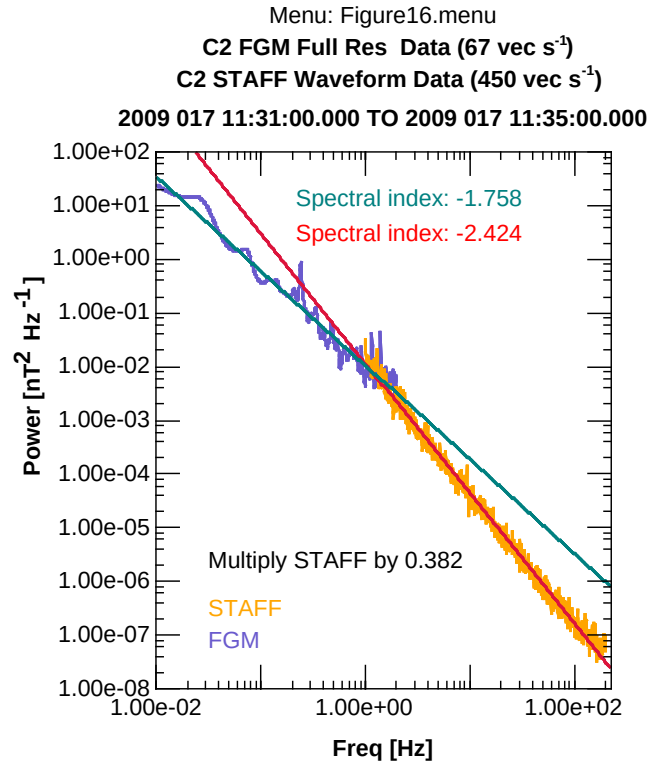

Figure 16. Magnetic field power density spectra during a period when there is no observed strahl. The spectra are formed by combining spectra from the full-resolution FGM data (blue portion of the spectra) and the STAFF waveform data (orange portion of the spectra). The two individual spectra are normalized between 1 and $2 \mathrm{~Hz}$.

(viz., Borovsky, 2008). This would provide for the narrow transition boundaries seen between the two plasma regimes. The supposition would place the flux tube boundary associated with the change in plasma state in Fig. 15 at about 23:56 UT. Figure 17 shows a plot of the electron plasma data and magnetic field. The plot is formatted almost identically to the plot shown in Fig. 9 but covers only a narrow slice of time centered on the flux tube crossing. In this figure, the data are gridded at $0.2 \mathrm{~s}$, which is the native time resolution of the magnetic field data used, while Fig. 9 is gridded at $4.0 \mathrm{~s}$, which is approximately the time resolution of the plasma data. Crossing the flux tube, there is a noticeable shift in both the average electron temperature and fluid velocity. Both of these can be traced to the existence or absence of the strahl. There are only very minor changes in the magnetic field across the flux tubes. This is no azimuthal rotation and only very small changes in the average elevation angle and average magnitude. Note that the apparent large azimuth rotation in Fig. 12 at this time is a rotation in flow as the strahl drops out of the eVDF and does not represent a rotation in the magnetic field.

\section{Conclusions}

In periods associated with slow wind $\left(\lesssim 425 \mathrm{~km} \mathrm{~s}^{-1}\right)$, there is often, but not always, no strahl observed in the solar wind or in the foreshock. It is not clear why the strahl is not seen
C2 LEEA MF moments

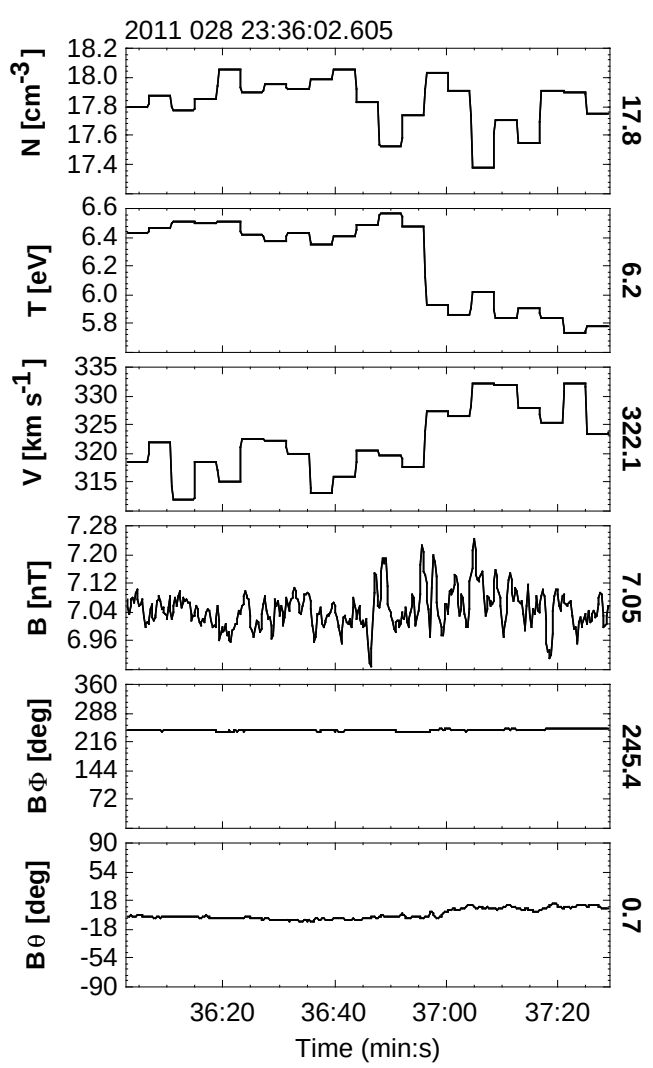

Figure 17. Identical to Fig. 9 but covering the narrow interval of time surrounding the transition between plasma with and without a strahl presence near 23:56 UT in Fig. 12. While there appears to be a large rotation in phase at this point in Fig. 12, what the figure really shows is a directional change in the flow as the strahl drops out of the eVDF. This occurs where the spacecraft might be transitioning between adjacent flux tubes.

in the eVDF during those slow solar wind intervals. Is the strahl simply not formed within the conditions under which the slow wind is set up or is it somehow disrupted at some time during or after its formation? It should be emphasized that these suppositions are based on what maybe considered a weak statistical study. There are insufficient observations during times when Cluster is upstream of the shock and returning data in burst-mode to accumulate a sufficient number of events to allow a full statistical analysis of the results. A statistical analysis will be presented in a follow-up paper if we are able to show that a similar analysis can be sufficiently carried out using the lower-resolution data that are commonly used in the solar wind. 


\section{Data availability}

With the exception of PEACE data, which were obtained from the Mullard Space Science Laboratory (MSSL) science data archive (http://www.mssl.ucl.ac.uk/missions/ cluster/about_peace_data.php), all data were obtained from the Cluster Science Archive (CSA, http://www.cosmos.esa. int/web/csa).

Acknowledgements. The authors would like to acknowledge the work and role of the Cluster Science Archive (CSA) and thank the EFW and FGM teams for providing the data used in this study. We would also like to acknowledge the PEACE team at MSSL who worked on and are constantly improving the instrument calibration. We thank the ACE SWEPAM instrument team and the ACE Science Center for providing the ACE data. We would like to acknowledge support from NASA Grant NNX15AI88G.

The topical editor, M. Temmer, thanks P. Yoon and three anonymous referees for help in evaluating this paper.

\section{References}

Anderson, B. R., Skoug, R. M., Steinberg, J. T., and McComas, D. J.: Variability of the solar wind suprathermal electron strahl, J. Geophys. Res., 117, A04107, doi:10.1029/2011JA017269, 2012.

Balogh, A., Dunlop, M. W., Cowley, S. W. H., Southwood, D. J., Thomlinson, J. G., Glassmeier, K. H., Musmann, G., Luhr, H., Buchert, S., Acuna, M. H., Fairfield, D. H., Slavin, J. A., Riedler, W., Schwingenschuh, K., and Kivelson, M. G.: The Cluster Magnetic Field Investigation, Space Sci. Rev., 79, 6591, doi:10.1023/A:1004970907748, 1997.

Borovsky, J. E.: Flux tube texture of the solar wind: Strands of the magnetic carpet at 1 AU?, J. Geophys. Res.-Space, 113, A08110, doi:10.1029/2007JA012684, 2008.

Che, H. and Goldstein, M. L.: The Origin of Non-Maxwellian Solar Wind Electron Velocity Distribution Function: Connection to Nanoflares in the Solar Corona, Astrophys. J. Lett., 795, L38, doi:10.1088/2041-8205/795/2/L38, 2014.

Che, H., Goldstein, M. L., and Viñas, A. F.: Bidirectional energy cascades and the origin of kinetic Alfvénic and whistler turbulence in the solar wind, Phys. Rev. Lett., 112, 061101, doi:10.1103/PhysRevLett.112.061101, 2014.

Cornilleau-Wehrlin, N., Chauveau, P., Louis, S., Meyer, A., Nappa, J. M., Perraut, S., Rezeau, L., Robert, P., Roux, A., de Villedary, C., de Conchy, Y., Friel, L., Harvey, C. C., Hubert, D., Lacombe, C., Manning, R., Wouters, F., Lefeuvre, F., Parrot, M., Pincon, J. L., Poirier, B., Kofman, W., and Louarn, P.: The Cluster SpatioTemporal Analysis of Field Fluctuations (STAFF) Experiment, Space Sci. Rev., 79, 107-136, doi:10.1023/A:1004979209565, 1997.

Cornilleau-Wehrlin, N., Mirioni, L., Robert, P., Bouzid, V., Maksimovic, M., de Conchy, Y., Harvey, C. C., and Santolík, O.: STAFF Instrument Products Distributed Through the Cluster Active Archive, The Cluster Active Archive, Studying the Earth's Space Plasma Environment, edited by: Laakso, H., Taylor, M. G. T. T., and Escoubet, C. P., Astrophysics and Space Science
Proceedings, Berlin: Springer, 11, 159-168, doi:10.1007/97890-481-3499-1_10, 2010.

Décréau, P. M. E., Fergeau, P., Krannosels'kikh, V., évêque, M. L., Martin, P. H., Randriamboarson, O., Sené, F. X., Trotignon, J. G., Canu, P., and Moögensen, P. B.: WHISPER, a resonance sounder and wave analyzer: Performances and persepctives for the Cluster mission, Space Sci. Rev., 79, 157-193, doi:10.1023/A:1004931326404, 1997.

Dum, C. T., Marsch, E., and Pilipp, W.: Determination of wave growth from measured distribution functions and transport theory, J. Plasma Phys., 23, 91-113, doi:10.1017/S0022377800022170, 1980.

Fazakerley, A. N., Lahiff, A. D., Wilson, R. J., Rozum, I., Anekallu, C., West, M., and Bacai, H.: PEACE Data in the Cluster Active Archive, The Cluster Active Archive, Studying the Earth's Space Plasma Environment, edited by: Laakso, H., Taylor, M. G. T. T., and Escoubet, C. P., Astrophysics and Space Science Proceedings, Berlin: Springer, 11, 129-144, doi:10.1007/97890-481-3499-1_8, 2010.

Feldman, W. C., Asbridge, J. R., Bame, S. J., Montgomery, M. D., and Gary, S. P.: Solar wind electrons, J. Geophys. Res., 80, 41814196, 1975.

Fitzenreiter, R. J., Ogilvie, K. W., Chornay, D. J., and Keller, J.: Observations of electron velocity distribution functions in the solar wind by the WIND spacecraft: High angular resolution strahl measurements, Geophys. Res. Lett., 25, 249-252, doi:10.1029/97GL03703, 1998.

Gary, S. P. and Saito, S.: Broadening of solar wind strahl pitch-angles by the electron/electron instability: Particlein-cell simulations, Geophys. Res. Lett., 34, L14111, doi:10.1029/2007GL030039, 2007.

Gary, S. P., Saito, S., and Li, H.: Cascade of whistler turbulence: Particle-in-cell simulations, Geophys. Res. Lett., 35, L02104, doi:10.1029/2007GL032327, 2008.

Gloag, J. M., Lucek, E. A., Alconcel, L.-N., Balogh, A., Brown, P., Carr, C. M., Dunford, C. N., Oddy, T., and Soucek, J.: FGM Data Products in the CAA, The Cluster Active Archive, Studying the Earth's Space Plasma Environment, edited by: Laakso, H., Taylor, M. G. T. T., and Escoubet, C. P., Astrophysics and Space Science Proceedings, Berlin: Springer, 11, 109-128, doi:10.1007/978-90-481-3499-1_7, 2010.

Gurgiolo, C., Goldstein, M. L., Viñas, A. F., and Fazakerley, A. N.: Direct observations of the formation of the solar wind halo from the strahl, Ann. Geophys., 30, 163-175, doi:10.5194/angeo-30163-2012, 2012.

Gustafsson, G., Bostrom, R., Holback, B., Holmgren, G., Lundgren, A., Stasiewicz, K., Ahlen, L., Mozer, F. S., Pankow, D., Harvey, P., Berg, P., Ulrich, R., Pedersen, A., Schmidt, R., Butler, A., Fransen, A. W. C., Klinge, D., Thomsen, M., Falthammar, C.G., Lindqvist, P.-A., Christenson, S., Holtet, J., Lybekk, B., Sten, T. A., Tanskanen, P., Lappalainen, K., and Wygant, J.: The Electric Field and Wave Experiment for the Cluster Mission, Space Sci. Rev., 79, 137-156, doi:10.1023/A:1004975108657, 1997.

Hammond, C. M., Feldman, W. C., McComas, D. J., and Forsyth, R. J.: Variation of electron-strahl width in the high-speed solar wind: ULYSSES observations, Astron. Astrophys., 316, 350354, 1996.

Johnstone, A. D., Alsop, C., Gurge, S., Carter, P. J., Coates, A. J., Coker, A. J., Fazakerley, A. N., Grande, M., Gowen, R. A., Gur- 
giolo, C., Hancock, B. K., Narheim, B., Preece, A., Sheather, P. H., Winningham, J. D., and Woodcliffe, R. D.: PEACE: A plasma electron and current experiment, Space Sci. Rev., 79, 351-398, 1997.

Khotyaintsev, Y., Lindqvist, P.-A., Eriksson, A., and André, M.: The EFW Data in the CAA, The Cluster Active Archive, Studying the Earth's Space Plasma Environment, edited by: Laakso, H., Taylor, M. G. T. T., and Escoubet, C. P., Astrophysics and Space Science Proceedings, Berlin: Springer, 11, 97-108, doi:10.1007/978-90-481-3499-1_6, 2010.

Lin, R. P.: Wind observations of suprathermal electrons in the interplanetary medium, Space Sci. Rev., 86, 61-78, 1998.

Maksimovic, M., Zouganelis, I., Chaufray, J.-Y., Issautier, K., Scime, E. E., Littleton, J. E., Marsch, E., McComas, D. J., Salem, C., Lin, R. P., and Elliott, H.: Radial evolution of the electron distribution functions in the fast solar wind between 0.3 and $1.5 \mathrm{AU}$, J. Geophys. Res.-Space, 110, 9104, doi:10.1029/2005JA011119, 2005.

Newbury, J. A., Russell, C. T., Phillips, J. L., and Gary, S. P.: Electron temperature in the ambient solar wind: Typical properties and a lower bound at $1 \mathrm{AU}, \mathrm{J}$. Geophys. Res., 103, 9553-9566, doi:10.1029/98JA00067, 1998.

Owens, M. J., Crooker, N. U., and Schwadron, N. A.: Suprathermal electron evolution in a Parker spiral magnetic field, J. Geophys. Res., 113, A11104, doi:10.1029/2008JA013294, 2008.

Pavan, J., Viñas, A. F., Yoon, P. H., Ziebell, L. F., and Gaelzer, R.: Solar Wind Strahl Broadening by Self-generated Plasma Waves, The Astrophys. J. Lett., 769, L30, doi:10.1088/20418205/769/2/L30, 2013.

Pierrard, V., Maksimovic, M., and Lemaire, J.: Core, halo, and strahl electrons in the solar wind, Astrophys. Space Sci., 277, 195-200, 2001.

Pierrard, V., Lazar, M., and Schlickeiser, R.: Evolution of the electric distribution function in whistler wave turbulence of the solar wind, Solar Phys., 269, 421-438, doi:10.1007/s11207-010-97007, 2011.

Pilipp, W. G., Miggenrieder, H., Montgomery, M. D., Mühlhaüser, K. H., Rosenbauer, H., and Schwenn, R.: Characteristics of electron velocity distribution functions in the solar wind derived from Helios Plasma Experiment, J. Geophys. Res., 92, 1075-1092, 1987a.

Pilipp, W. G., Miggenrieder, H., Mühlhaüser, K. H., Rosenbauer, H., Schwenn, R., and Neubauer, F. M.: Variations of electron velocity distribution functions in the solar wind, J. Geophys. Res., 92, 1103-1118, 1987b.

Rosenbauer, H., Miggenrieder, H., Montgomery, M. D., and Schwenn, R.: Preliminary results of the Helios plasma experiment, in: Physics of Solar Planetary Environments, edited by: Williams, D. J., p. 319, American Geophysical Union, Washington DC, USA, 1976.
Rosenbauer, H., Schwenn, R., Marsch, E., Meyer, B., H, M., Montgomery, M. D., Muhlhausser, K. H., Pilipp, W., Voges, W., and Zink, S. M.: A survey on initial results of the Helios plasma experiment, J. Geophys., 42, 561-580, 1977.

Saito, S. and Gary, S. P.: Whistler scattering of suprathermal electrons in the solar wind: Particle-in-cell simulations, J. Geophys. Res., 112, A06116, doi:10.1029/2006JA012216, 2007a.

Saito, S. and Gary, S. P.: All whistlers are not created equally: Scattering of strahl electrons in the solar wind via particle-in-cell simulations, Geophys. Res. Lett., 34, L01102, doi:10.1029/2006GL028173, 2007b.

Seough, J., Nariyuki, Y., Yoon, P. H., and Saito, S.: Strahl Formation in the Solar Wind Electrons via Whistler Instability, Astrophys. J. Lett., 811, L7, doi:10.1088/2041-8205/811/1/L7, 2015.

Smith, H. M., Marsch, E., and Helander, P.: Electron Transport in the Fast Solar Wind, Astrophys. J., 753, 31, doi:10.1088/0004637X/753/1/31, 2012.

Stverák, V. S. V., Maksimovic, M., Trávníčeik, P., Marsch, E., Fazakerley, A. N., and Scieme, E. E.: Radial evolution of nonthermal electron populations in the low-latitude solar wind: Cluster and Ulysses observations, J. Geophys. Res., 114, A05104, doi:10.1029/2008JA013883, 2009.

Trotignon, J. G., Décréau, P. M. E., Rauch, J. L., Vallières, X., Rochel, A., Kougblénou, S., Lointier, G., Facskó, G., Canu, P., Darrouzet, F., and Masson, A.: The WHISPER Relaxation Sounder and the CLUSTER Active Archive, The Cluster Active Archive, Studying the Earth's Space Plasma Environment, edited by: Laakso, H., Taylor, M. G. T. T., and Escoubet, C. P., Astrophysics and Space Science Proceedings, Berlin: Springer, 2010, 11, 185-208, doi:10.1007/978-90-481-3499-1_12, 2010.

Viñas, A. F., Gurgiolo, C., Nieves-Chinchilla, T., Gary, S. P., and Goldstein, M. L.: Whistler waves driven by anisotropic 3D strahl velocity distributions in the solar wind: Cluster observations, in: AIP Proceedings of Solar Wind 12 Conference, edited by: Maksimovic, M., Issautier, K., Meyer-Vernet, N., Moncuquet, M., and Pantellini, F., p. 265, American Institute of Physics, New York, NY, USA, 2010.

Vocks, C.: Kinetic Models for Whistler Wave Scattering of Electrons in the Solar Corona and Wind, Space Sci. Rev., 172, 303314, doi:10.1007/s11214-011-9749-0, 2012.

Vocks, C., Mann, G., and Rausche, G.: Formation of suprathermal electron distributions in the quiet solar corona, Astron. Astrophys., 480, 527-536, doi:10.1051/0004-6361:20078826, 2008.

Wang, L., Lin, R. P., Salem, C., Pulupa, M., Larson, D. E., Yoon, P. H., and Luhmann, J. G.: Quiet-time Interplanetary 2-20 keV Superhalo Electrons at Solar Minimum, Astrophys. J. Lett., 753, L23, doi:10.1088/2041-8205/753/1/L23, 2012. 